EPJ Web of Conferences 49, 17008 (2013)

DOI: $10.1051 /$ epjconf/20134917008

(C) Owned by the authors, published by EDP Sciences, 2013

\title{
Measurement of the Top Quark Properties with the ATLAS Detector
}

\author{
Stefan Guindon ${ }^{1,2}$, a , On behalf of the ATLAS Collaboration. \\ ${ }^{1}$ II Physikalisches Institut, Georg-August-Universität Göttingen, Friedrich-Hund-Platz 1 Göttingen, D-37077 Germany \\ ${ }^{2}$ University at Albany, 1400 Washington Ave. Albany NY, 12222 United States of America
}

\begin{abstract}
The top quark, discovered at the Tevatron in 1995 by the CDF and D $\varnothing$ collaborations, is the heaviest known particle. The measurement of its properties are of interest to the understanding of the strong and weak forces, as well as searches for physics beyond the Standard Model. Several measurements of top quark properties are presented using data collected at the ATLAS experiment located at the Large Hadron Collider (LHC) at $\sqrt{s}=7 \mathrm{TeV}$ centre-of-mass energy. The measurements include top quark polarisation, spin correlation, production of $t \bar{t} Z$ and $t \bar{t} \gamma$, as well as top mass and $W t b$ vertex measurements in both the lepton + jets and dilepton channel.
\end{abstract}

\section{Introduction}

The top quark was discovered in 1995 at the Tevatron by the CDF and DØ collaborations [1]. Since the top quark is so heavy, it decays rapidly to a $W$ boson and a $b$-quark. Since the decay occurs before hadronization $\left(O\left[10^{-24} s\right]\right)$, the top quark properties are directly accessible through its decay products [2]. As a result, top quark properties measurements give a unique opportunity to study a bare quark. Studies of the top quark properties play a vital role in the understanding of the Standard Model (SM) and to possible hints of new physics.

In top pair decays, the further decay of the $W$ boson determines in which type of decay channel the top quark is classified. The $W$ can either decay leptonically or hadronically. Therefore, when a top quark pair $t \bar{t}$ is produced, if both top quarks decay leptonically, the channel is declared to be dilepton. This channel contains two high $p_{\mathrm{T}}$ leptons (either $e$ or $\mu$ from $W$ or $\tau$ decays), two $b$-jets and a significant portion of missing transverse energy from the two neutrinos. If both $W$ bosons decay hadronically, the channel is classified as all-hadronic. In this channel, six jets are expected, at least two of which are $b$-jets. Finally, if one of each $W$ boson decay is observed, the channel is stated to be lepton + jets. This channel contains one high $p_{\mathrm{T}}$ lepton, four jets, again at least two of which are $b$-jets, and some missing transverse energy due to the escaping neutrino. All of the analyses presented here are in the lepton + jets and dilepton decay channels.

\section{The ATLAS Experiment}

The ATLAS experiment [3] is one of four large experiments located along the Large Hadron Collider

\footnotetext{
a e-mail: stefan.guindon@cern.ch
}

(LHC) at CERN in Geneva, Switzerland. Two proton beams were made to collide at the centre of the detector with a centre-of-mass energy of $7 \mathrm{TeV}$ during 2011 data-taking. The detector is comprised of three sub-detector groups. The innermost part of the detector, the inner detector, consists of several pixel layers followed by a silicon strip tracker and a transition-radiation tracker. The second part of the detector comprises two calorimeters (electromagnetic and hadronic calorimeters). The outermost part of the detector is the muon spectrometer used for measurements of muon momentum and position.

The dataset used in the analyses was collected at the ATLAS Experiment during the $2011 \sqrt{s}=7 \mathrm{TeV}$ run. The total luminosity contains up to $4.7 \mathrm{fb}^{-1}$ of data, depending on the analysis.

\section{Top Quark Properties}

\subsection{Kinematic Likelihood Reconstruction}

The measurements of the top quark properties rely on information from the decay products of the $t \bar{t}$ system, whether it is the angular distributions or the masses of the hadronic and leptonic top quark hemispheres. If the jets from the $t \bar{t}$ decay are not properly assigned, the measurements of these properties are much more difficult. As a result, a large emphasis is placed on properly assigning the jets from the $t \bar{t}$ decay to the corresponding quarks.

A kinematic likelihood fitter (KLFitter) is used to improve the probability of properly assigning the jet to its decay particle. The probability of properly assigning all jets is increased to $70 \%$ or higher. This is done using Breit-Wigner functions to constrain the measured masses of the two $W$ bosons, as well as the two reconstructed top quarks. In the fit, the masses of the two top hemispheres are required to be identical. Transfer functions were derived using Monte 
Carlo (MC) and are used to correct for detector effects as well as influences due to hadronization. The likelihood function is written in Ref. [4] along with an example of the transfer functions used.

Not only is the kinematic information used, but also $b$-tagging information is employed to reduce the number of possible $b$-jet assignments in the event by requiring them to match a $b$-quark in the model. The KLFitter is used in the top quark polarisation, top mass and $W t b$ vertex measurements presented.

\subsection{Top Quark Polarisation}

Since the top quark decays so quickly, the polarisation can be measured directly from the decay products. Measurements of the top quark polarisation may give hints of physics beyond the SM. The top quark is expected to be unpolarised in the SM due to parity conservation in QCD, however some beyond SM models predict top quark production involving positive or negative polarisation [5].

To measure the top quark polarisation, the polar angle of the lepton as measured in the rest frame of its parent top quark $\left(\cos \left(\theta_{l}\right)\right)$ is used in the lepton + jets channel [6] with an integrated luminosity of $4.7 \mathrm{fb}^{-1}$. The KLFitter described in Sec 3.1 is used to properly reconstruct the $t \bar{t}$ system. From there, a template fit of the polar angle is used to measure the polarisation. The $\cos \left(\theta_{l}\right)$ distribution is shown in Fig. 1 and compared to no polarisation, positive, and negative polarisation.

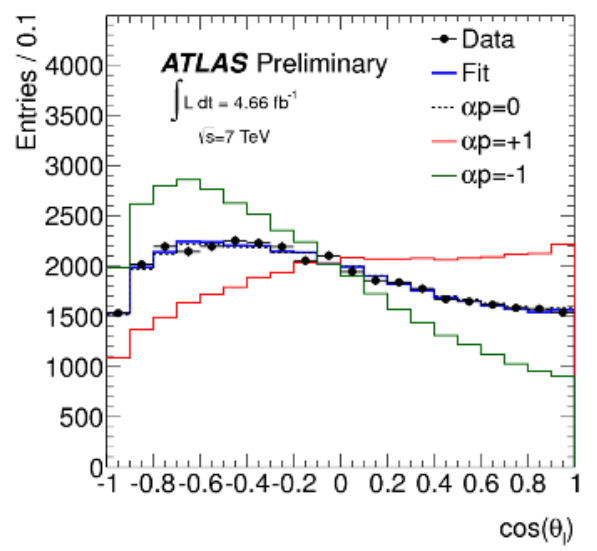

Figure 1. Reconstructed $\cos \left(\theta_{l}\right)$ distributions for fully, partially and negatively polarised top quarks. The three templates are shown with the $\mu+$ jets data [6].

From the polar angle, the fraction of positively charged quarks can be written as:

$$
f=\frac{1}{2}+\frac{N\left(\cos \theta_{l}>0\right)-N\left(\cos \theta_{l}<0\right)}{N\left(\cos \theta_{l}>0\right)+N\left(\cos \theta_{l}<0\right)} .
$$

The degree of polarisation is written as $\alpha p=$ $2 f-1$. The fraction of positively charged top quarks is measured to be: $f=0.470 \pm 0.009$ (stat.) ${ }_{-0.032}^{+0.023}$ (syst.), which corresponds to a degree of polarisation of $-0.060 \pm 0.018$ (stat. $)_{-0.064}^{+0.046}$ (syst.) of top quarks. This is consistent with the SM expected value of no polarisation. The largest sources of systematics are from jet resolution and calibration scales.

\subsection{Spin Correlation}

As in the polarisation measurement, the spin correlation of top quarks may give hints of physics beyond the SM. Though top quarks are produced unpolarised, their spins are expected to be correlated [7]. The observed degree of correlation $A$, can be written as follows:

$$
A=\frac{N(\uparrow \uparrow)+N(\downarrow \downarrow)-N(\uparrow \downarrow)-N(\downarrow \uparrow)}{N(\uparrow \uparrow)+N(\downarrow \downarrow)+N(\uparrow \downarrow)+N(\downarrow \uparrow)} .
$$

$N(\uparrow \uparrow)$ represents the number of events with parallel spin states, whereas $N(\uparrow \downarrow)$ represents anti-parallel spin states. The strength of $A$ depends on both the production mechanism, which is $g g$-fusion dominated at the LHC, and also the choice of a quantization axis.

In the dilepton channel using $2.1 \mathrm{fb}^{-1}$ of data, this degree of correlation is measured via a fit of the measured distribution of the angle between the two leptons $(\Delta \phi)$, shown in Figure 2.

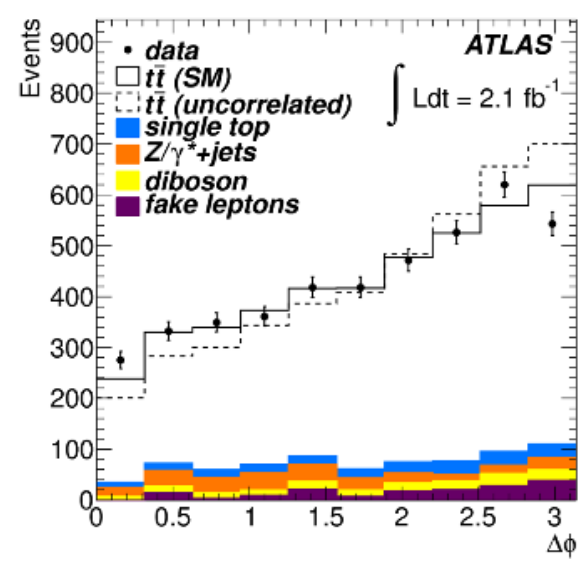

Figure 2. Correlated and uncorrelated top MC with stacked background expectation compared to data using the angle between two leptons $(\Delta \phi)$ in the dilepton channel [8].

Fits are converted into both the helicity basis $\left(A_{\text {hel }}\right)$ and maximal basis $\left(A_{\max }\right)$ [8]. The helicity basis uses the direction of flight of the top quark in the center-of-mass frame of the $t \bar{t}$-system. The maximal basis is optimized for $g g \rightarrow t \bar{t}$ production [9]. The template fit compares templates of correlated $\left(f^{\mathrm{SM}}=1\right)$ and uncorrelated $\left(f^{\mathrm{SM}}=0\right)$ spins. The fit is performed in all three dilepton channels simulataneously and yields: $f^{\mathrm{SM}}=1.30 \pm$ 0.14 (stat.) $)_{-0.22}^{+0.27}$ (syst.). This is translated to the two spin bases: $A_{\text {hel }}=0.40 \pm 0.04$ (stat.) ${ }_{-0.07}^{+0.08}$ (syst.), and $A_{\max }=0.57 \pm 0.06$ (stat. $)_{-0.10}^{+0.12}$ (syst.).

The SM expected values in these two spin bases are: $A_{\text {hel }}=0.31$ and $A_{\max }=0.44$, with theoretical uncertainties of the order of $1 \%$. This is the first observation of $t \bar{t}$ spin correlation with a significance of $5.1 \sigma$. 


\subsection{Search for $t \bar{t} Z$ Production}

The search for $t \bar{t} Z$ is the search for the production of two top quarks along with the $Z$ boson. The search was performed in the $t \bar{t} \rightarrow$ lepton + jets channel where two extra leptons compatible with a $Z$ boson decay are required in the selection. The full 2011 dataset was used in the measurement, containing $4.7 \mathrm{fb}^{-1}[10]$.

The expected number of signal events is $0.85 \pm$ 0.04 (stat.) \pm 0.14 (syst.) with a background expectation of $0.28 \pm 0.05$ (stat.) \pm 0.14 (syst.). The largest uncertainty is derived from the background MC expectation, which is set at $50 \%$. The fake lepton estimate was performed using the matrix method [11]. The signal and background expectations are shown for the mass of the two oppositely charged leptons in Figure 3 along with the one data candidate event found in the selection. The leptons mass should be a result of the $Z$ decay.

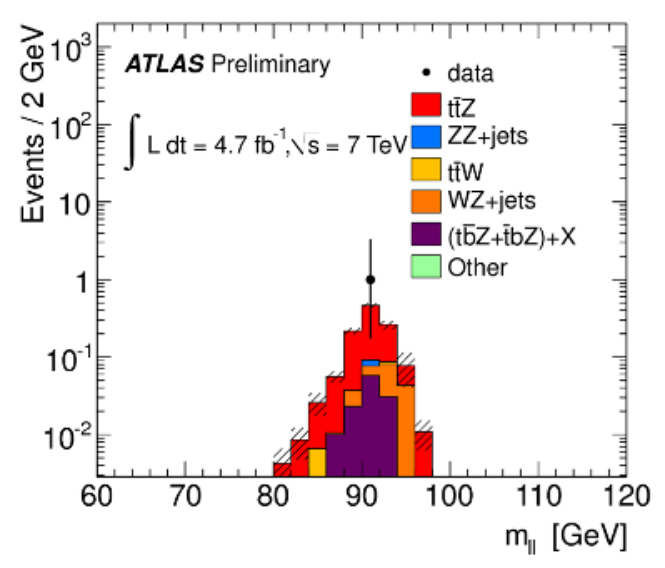

Figure 3. Distribution for the mass of the two oppositely charged leptons for the single candidate $t \bar{t} Z$ event compared to the expected background [10].

The data event contains two oppositely charged muons compatible with coming from the $Z$ boson and one electron from the top decay. From the single event, a $95 \%$ C.L. limit on the observed $t \bar{t} Z$ cross section can be placed at: $\sigma_{t \bar{t} Z}<0.71 \mathrm{pb}$.

\section{$3.5 t \bar{t} \gamma$ Cross-section Measurement}

The measurement of the $t \bar{t} \gamma$ cross-section was performed using $1.04 \mathrm{fb}^{-1}$ of the 2011 dataset [12] in the lepton + jets channel. The search was done using a template method, where templates were created for signal $t \bar{t} \gamma$ and backgrounds from non- $t \bar{t}$ background, $t \bar{t} \gamma$ background, electron and hadron fakes. The templates are created as a function of the isolation value

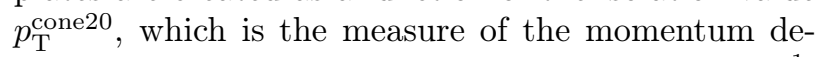
posited around the object with a radius of $\Delta R=0.2^{1}$, a function of the pseudo-rapidity $(\eta)$ and azimuthal angle $(\phi)$. This can be seen in Figure 4.

The template method distinguishes prompt photons from hadron fakes. Low values of $p_{\mathrm{T}}^{\text {cone20 }}$ represent isolated photons. It is expected most signal

\footnotetext{
${ }^{1} \Delta R=\sqrt{\Delta \eta^{2}+\Delta \phi^{2}}$
}

photon candidates should be isolated, whereas hadron fakes have a flat expectation in $p_{\mathrm{T}}^{\text {cone20 }}$. Backgrounds are determined through data-driven models. The templates are also $p_{\mathrm{T}}$ dependent.

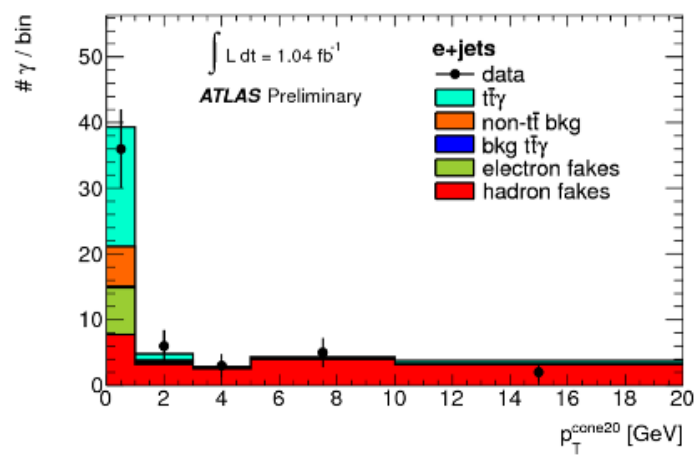

Figure 4. Isolation templates as a function of $p_{T}^{\text {cone20 }}$ for signal $t \bar{t} \gamma$ and several background contributions plotted with the observed data in the $e+$ jets channel [12].

The cross section is determined to be: $\sigma_{t \bar{t} \gamma}=$ $2.0 \pm 0.50$ (stat.) \pm 0.70 (syst.) \pm 0.08 (lumi.) pb for photons with $p_{\mathrm{T}}>8 \mathrm{GeV}$. The largest systematic uncertainties come from initial and final state radiation, as well as the jet energy scale and photon identification. This cross section corresponds to a $2.7 \sigma$ significance.

\subsection{Top Mass Measurements}

The top quark mass is already the most precisely known quark mass from measurements made at the Tevatron [13]. The mass measurements presented in this note were performed in both lepton + jets and dilepton channels.

\subsubsection{Lepton + Jets Mass Measurement}

The top mass was measured in the lepton + jets channel using two separate measurements with $1.04 \mathrm{fb}^{-1}$ of data [14]. Both measurements perform a template fit comparing signal and background expectations to data. The first measurement uses a 2-dimensional fit of both the top mass and $W$ mass. The simultaneous measurement of the top and $W$ mass allows for a measure of the jet energy scale, one of the largest systematic uncertainties on the top mass measurement.

The measurement was made in the hadronic top hemisphere, where a $b$-jet was assigned to a pair of light jets within a reconstructed mass window of $[50,100] \mathrm{GeV}$. The jet triplet assignment with the highest $p_{\mathrm{T}}$ is taken as the decay products from the top quark. A $\chi^{2}$ likelihood is used to correct the energies of the hadronic top quark due to detector and hadronization effects. The reconstructed hadronic top mass decay is shown in Figure 5. The top mass is parametrized using the sum of a Gaussian and Landau function.

The second top quark mass measurement in the lepton + jets channel uses the full $t \bar{t}$ decay system in the KLFitter, as shown in Sec 3.1. A $R_{32}$ variable is 
created to perform a per-event jet energy scale calibration in the 1-dimensional fit. The $R_{32}$ variable is defined as: $R_{32}=\frac{m_{\text {top }}^{\text {reco }}}{m_{\mathrm{W}}^{\text {reco }}}$. Both top and $W$ masses are reconstructed from the hadronic hemisphere. The performance of the variable can be seen in Refs. [4, 15]. The reconstructed $R_{32}$ variable is seen in Figure 6 . The template fit is made to the $R_{32}$ variable, which is dependent on the top mass.

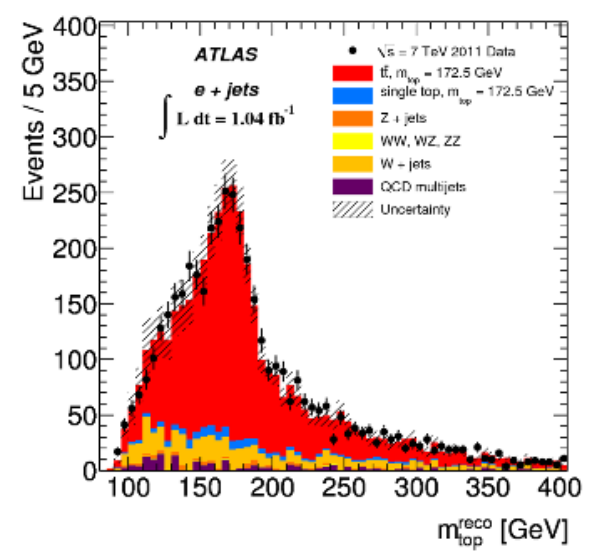

Figure 5. Reconstructed hadronic top mass for signal and background MC expectations compared to the measured data. The fit is made simultaneously to the top mass peak and the reconstructed $W$ boson mass [14].

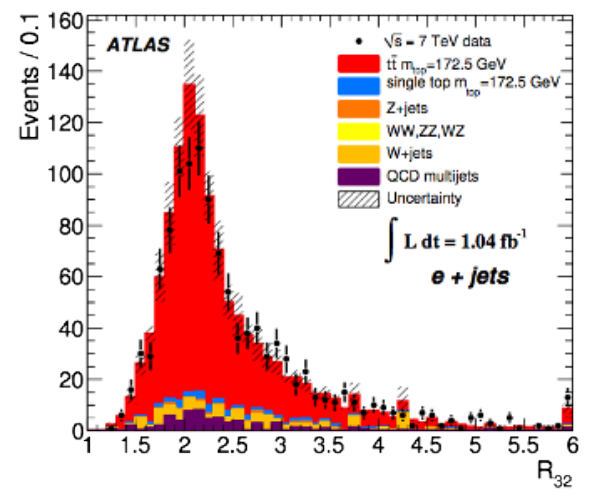

Figure 6. Reconstructed $R_{32}$ top mass estimator used in the 1-dimendional top mass measurement. The signal and background MC estimates are compared to data [14].

In the 1-dimensional analysis, additional selection on the likelihood output, as well as stricter $p_{\mathrm{T}}$ cuts are employed to reduce systematic uncertainties arising from the jet energy scale and $b$-jet energy scale. As a result, there is a significant reduction in the number of events analyzed in the 1-dimentional (1d) analysis compared to the 2-dimensional (2d) analysis.

The top mass measurements in the 2 - $\mathrm{d}$ and 1 -d analyses are:

$$
\begin{aligned}
& m_{\text {top }}^{2 \mathrm{~d}}=174.5 \pm 0.6 \text { (stat.) } \pm 2.3 \text { (syst.) } \mathrm{GeV}, \\
& m_{\text {top }}^{1 \mathrm{~d}}=174.4 \pm 0.9 \text { (stat.) } \pm 2.5 \text { (syst.) } \mathrm{GeV} .
\end{aligned}
$$

The ATLAS top mass measurement is taken from the 2-dimensional analysis. The largest systematic uncertainties result from initial and final state radiation, as well as the jet and $b$-jet energy scales. These three uncertainties account for almost $90 \%$ of the total uncertainty on the top mass measurement.

\subsubsection{Dilepton Mass Measurement}

The top mass was also measured with the ATLAS experiment in the $e \mu$ dilepton channel using the full 2011 dataset of $4.7 \mathrm{fb}^{-1}$ [16]. In the dilepton channel, the top quarks cannot be reconstructed due to underconstrained kinematics resulting from the two escaping neutrinos. As a result, the mass is estimated using the $m_{\mathrm{T} 2}$ variable [17].

The $m_{\mathrm{T} 2}$ variable assumes two invisible particles in the decay chain along with the reconstructed particles. It is written as:

$m_{\mathrm{T} 2}=\min _{p_{\mathrm{T}}^{(i))}}\left\{\max \left[m_{\mathrm{T}}\left(m_{\mathrm{invis}}, p_{\mathrm{T}}^{(1)}\right), m_{\mathrm{T}}\left(m_{\mathrm{invis}}, p_{\mathrm{T}}^{(2)}\right)\right]\right\}$, where each particle $m_{\mathrm{T}}$ is defined as:

$m_{\mathrm{T}}=\sqrt{m_{\mathrm{vis}}^{2}+m_{\text {invis }}^{2}+2\left(E_{\mathrm{T}}^{\mathrm{vis}} E_{\mathrm{T}}^{\text {invis }}-p_{\mathrm{T}}^{\mathrm{vis}} \cdot p_{\mathrm{T}}^{(i)}\right)}$.

The variable gives an upper bound on the parent particle mass, in this case the top mass. This can be seen in Figure 7.

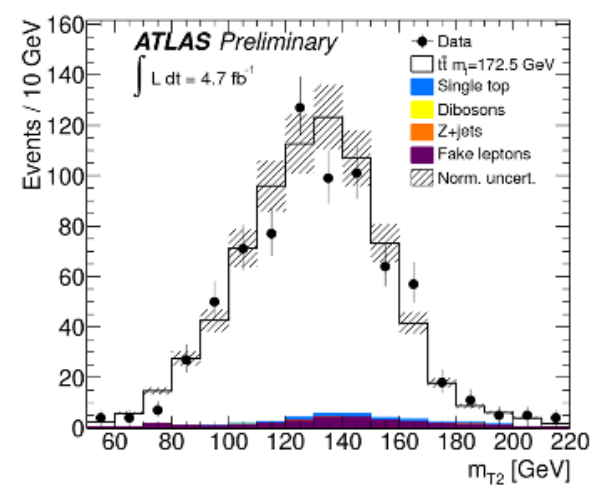

Figure 7. Reconstructed $m_{\mathrm{T} 2}$ distribution in the $e \mu$ channel for signal and background expectation compared to data. The variable reconstructs the parent top quark mass with two invisible neutrinos in the decay chain [16].

A template analysis is performed using di ff erent mass points, where the fit is made to the mean of the reconstructed $m_{\mathrm{T} 2}$ distribution. The resulting top mass is measured to be: $m_{\text {top }}=175.2 \pm$ 1.6(stat.) ${ }_{-2.8}^{+3.1}$ (syst.) $\mathrm{GeV}$. The largest uncertainties arise from jet and $b$-jet energy scale, as in the lepton + jets channel measurement, and the generator uncertainty used for the signal MC.

\section{7 $W t b$ Vertex Measurement}

The top quark decay vertex, also written as $W t b$ vertex, follows the $V-A$ structure of weak interactions, where $V$ and $A$ represent vertex and axialvector components. The fraction of longitudinal $\left(F_{0}\right)$, left-handed $\left(F_{\mathrm{L}}\right)$ and right-handed $\left(F_{\mathrm{R}}\right) W$ boson helicity states can be expressed in terms of the angular distribution of the top quark decay products: $\frac{1}{\sigma} \frac{d \sigma}{d \cos \theta^{*}}=\frac{3}{4}\left(1-\cos ^{2} \theta^{*}\right) F_{0}+\frac{3}{8}\left(1-\cos \theta^{*}\right)^{2} F_{\mathrm{L}}+\frac{3}{8}\left(1+\cos \theta^{*}\right)^{2} F_{\mathrm{R}}$.

It is expected from NNLO QCD calculations that $F_{0}=0.687 \pm 0.005, F_{\mathrm{L}}=0.311 \pm 0.005$, and $F_{\mathrm{R}}=$ 
$0.0017 \pm 0.0001$ [18]. The $\cos \theta^{*}$ distribution is measured by taking the angle between lepton and reverse $b$ quark direction in the $W$ rest frame.

The helicity fractions are measured in two ways, in both the lepton + jets and dilepton channels using $1.04 \mathrm{fb}^{-1}$ [19]. The first measurement performs a template fit of the sum of the three helicity states to the $\cos \theta^{*}$ distribution. The templates were produced with the Protos MC generator [20], each with a longitudinal, left-, and right-handed helicity of 1 . The fit to data is shown in Figure 8 in the lepton + jets channel, where the SM expectation of the helicity states is compared to the best fit to data along with the uncertainties. In the lepton + jets channel, the KLFitter was also used for the kinematic reconstruction.

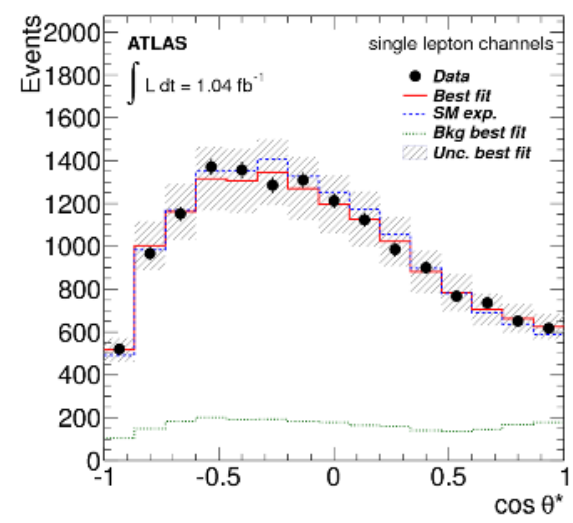

Figure 8. Distribution of the reconstructed $\cos \theta^{*}$ in the lepton + jets channel. The figure shows the sum of signal and background expectation (dashed line) compared with the best fit from data (solid) [19].

The second measurement performed in both lepton + jets and dilepton channel is the asymmetries measurement. The angular asymmetries can be written as:

$$
A_{ \pm}=\frac{N\left(\cos \theta^{*}>\mathrm{z}\right)-\mathrm{N}\left(\cos \theta^{*}<\mathrm{z}\right)}{N\left(\cos \theta^{*}>\mathrm{z}\right)+\mathrm{N}\left(\cos \theta^{*}<\mathrm{z}\right)} .
$$

When $z= \pm\left(1-2^{2 / 3}\right)$, the dependence of $F_{\mathrm{L}}$ on $F_{\mathrm{R}}$ is cancelled. The measurement is then transformed into the helicity fractions.

The four measurements were combined into one ATLAS measurement. The results were combined using the BLUE method [21], and are to-date the most precise measurement of the helicity fractions. The results are also compatible with the SM expectation. The resulting helicity fractions are measured to be $F_{0}=0.67 \pm 0.03$ (stat.) \pm 0.06 (syst.), $F_{\mathrm{L}}=0.32 \pm 0.02$ (stat.) \pm 0.03 (syst.), and $F_{\mathrm{R}}=$ $0.01 \pm 0.01$ (stat.) \pm 0.04 (syst.). The largest uncertainties are from initial and final state radiation, as well as the jet energy scale in the template method and background normalization in the asymmetries measurements.

\section{Conclusions}

The 2011 dataset collected with the ATLAS detector was used to measure many top quark properties at $\sqrt{s}=7 \mathrm{TeV}$ energies. They include the first observation of spin correlation as well as the most precise helicity fractions measurement. Measurements of the top mass were performed in both lepton + jets and dilepton decay channels, both in agreement with previous measurements at the Tevatron. Furthermore, this dataset enabled the first measurement of the $t \bar{t} \gamma$ cross-section with a $2.7 \sigma$ significance and a search for $t \bar{t} Z$ production. Finally, the top quark polarisation was also measured to be compatible with the SM expectation.

Most of the measurements are now dominated by systematic uncertainties. A large portion of the uncertainty comes from the jet and $b$-jet energy scales. Over the next several years, measurements in the top quark sector will be highlighted by more precise determinations of the properties through better understanding the ATLAS detector.

\section{References}

[1] CDF Collaboration, F. Abe et al., Phys. Rev. Lett. 74, 2626 (1995); DØ Collaboration, S. Abachi et al., Phys. Rev. Lett. 74, 2632 (1995).

[2] I. Bigi, Y. Dokshitzer, V. Khoze, J. Kühn, and P. Zerwas, Phys. Lett. B 181, 157 (1986).

[3] ATLAS Collaboration, 2008 JINST 3 S08003.

[4] ATLAS Collaboration, ATLAS-CONF-2011-033, (2011). http://cdsweb.cern.ch/record/1337783.

[5] R. Frederix and F. Maltoni, JHEP 01, 047 (2009).

[6] ATLAS Collaboration, ATL-CONF-2012-133, (2012). http://cdsweb.cern.ch/record/1478373.

[7] W. Bernreuther, A. Brandenburg, Z. G. Si, and P. Uwer, Phys. Rev. Lett. 87, 242002 (2001).

[8] ATLAS Collaboration, Phys. Rev. Lett. 108, 212001 (2012).

[9] P. Uwer, Phys. Lett. B 609, 271 (2005).

[10] ATLAS Collaboration, ATLAS-CONF-2012-126, (2012). http://cdsweb.cern.ch/record/1474643.

[11] DØ Collaboration, Phys. Rev. D 76 (2007).

[12] ATLAS Collaboration, ATLAS-CONF-2011-153, (2011). http://cdsweb.cern.ch/record/1398197.

[13] The CDF and D $\varnothing$ Collaborations and the Tevatron Electroweak Working Group, arxiv:1107.5255 (2011).

[14] ATLAS Collaboration, Eur.Phys.J. C72 (2012) 2046.

[15] ATLAS Collaboration, ATL-PHYS-PUB-2010004, (2010). https://cds.cern.ch/record/1267950.

[16] ATLAS Collaboration, ATL-CONF-2012-082, (2012). http://cdsweb.cern.ch/record/1460394.

[17] CDF Collaboration, Phys. Rev. D81 (2010).

[18] A. Czarnecki, J.G. Korner and J.H. Piclum, Phys. Rev. D 81, 111503 (2010).

[19] ATLAS Collaboration, JHEP 1206 (2012) 088.

[20] J. Aguilar-Saavedra, et. al., Eur. Phys. J. C50, 519 (2007).

[21] L. Lyons, D. Gibaut, and P. Clifford, Nucl. Instr. and Meth., A270, 1988, (110). 